\title{
WEIGHT FORMULAS FOR TERNARY MELAS CODES
}

\author{
GERARD VAN DER GEER, RENÉ SCHOOF, AND MARCEL VAN DER VLUGT
}

\begin{abstract}
In this paper we derive a formula for the frequencies of the weights in ternary Melas codes and we illustrate this formula by computing a table of examples.
\end{abstract}

\section{INTRODUCTION}

Let $q=p^{m}$, where $p$ is a prime, and let $\alpha$ be a generator of the multiplicative group $\mathbf{F}_{q}^{*}$. Consider the cyclic code $C$ over $\mathbf{F}_{q}$ of length $q-1$ with generator polynomial $(X-\alpha)\left(X-\alpha^{-1}\right)$. The dual code $C^{\perp}$ is cyclic with zeros $1, \alpha^{2}, \alpha^{3}, \ldots, \alpha^{q-3}$, which are zeros of the polynomials

$$
\sum_{i=0}^{q-2}\left(a \alpha^{i}+b \alpha^{-i}\right) X^{i} \in \mathbf{F}_{q}[X] /\left(X^{q-1}-1\right) \quad \text { with } a, b \in \mathbf{F}_{q} .
$$

This implies that the code

$$
D=\left\{(a x+b / x)_{x \in \mathbf{F}_{q}^{*}}: a, b \in \mathbf{F}_{q}\right\}
$$

satisfies $D=C^{\perp}$. The classical Melas code $M(q)$ is defined as the restriction to $\mathbf{F}_{p}$ of the code $C$ (see [5, 4]). By Delsarte's theorem [4, p. 208] we have

$$
\operatorname{Tr}\left(C^{\perp}\right)=\left(\left.C\right|_{\mathbf{F}_{p}}\right)^{\perp},
$$

where $\operatorname{Tr}$ is the trace map from $\mathbf{F}_{q}$ to $\mathbf{F}_{p}$. If we substitute $C^{\perp}=D$ and $\left.C\right|_{\mathbf{F}_{p}}=M(q)$ in Delsarte's theorem, we find

$$
\left\{(\operatorname{Tr}(a x+b / x))_{x \in \mathbf{F}_{q}^{*}}: a, b \in \mathbf{F}_{q}\right\}=M(q)^{\perp} .
$$

To ensure injectivity of the trace map, we require $2 m+1<q$. Then the dual code $M(q)^{\perp}$ has dimension $2 m$.

In $[6,1]$ we determined the weight distribution of $M(q)^{\perp}$ for $p=2$ and 3. Then, by the MacWilliams identities and the Eichler-Selberg trace formula we derived a formula for the number $A_{i}$ of code words of weight $i$ in $M(q)$ involving traces of Hecke operators on certain spaces of cusp forms [6, Theorem 4.2; 1 , Theorem 2.3]. Especially for $p=3$, this was done in a rather concise way, only announcing results and further illustrations. In this paper we will work out the case $p=3$ and illustrate the result by computing some weight formulas for ternary Melas codes.

Received December 21, 1989; revised March 4, 1991.

1991 Mathematics Subject Classification. Primary 94B05, 11F11.

The second author was supported by the Netherlands Organization of Scientific Research. 
An outline of this paper is as follows. In $\S 2$ we derive an expression for traces of Hecke operators on $S_{k}\left(\Gamma_{1}(3)\right)$. In $\S 3$ we prove the weight distribution theorem for ternary Melas codes. Then, in the next sections, we compute traces of Hecke operators, first for even $k$, then for odd $k$. Finally, in $\S 6$ we give a table of weight formulas for $M(q)$.

The references on coding theory can be found in the book of MacWilliams and Sloane [4]. For a systematic introduction to cusp forms and Hecke operators we refer to the books by S. Lang [2] and J.-P. Serre [8]. In [6, Theorem 2.2] the reader can find the precise form of the Eichler-Selberg trace formula, as we use it. Our notation in this paper links up with the notation in [6].

\section{Traces of Hecke operators on $S_{k}\left(\Gamma_{1}(3)\right)$}

For the space of cusp forms $S_{k}\left(\Gamma_{1}(3)\right)$ we have

$$
S_{k}\left(\Gamma_{1}(3)\right)=S_{k}\left(\Gamma_{0}(3), 1\right) \oplus S_{k}\left(\Gamma_{0}(3), \omega\right),
$$

where 1 is the trivial character on $(\mathbf{Z} / 3 \mathbf{Z})^{*}$ and $\omega$ is the quadratic character on $(\mathbf{Z} / 3 \mathbf{Z})^{*}$. Both characters have conductor 3 , and we extend them to $\mathbf{Z} / 3 \mathbf{Z}$ by defining them 0 on the residue class of 0 modulo 3 . Actually,

$$
S_{k}\left(\Gamma_{1}(3)\right)= \begin{cases}S_{k}\left(\Gamma_{0}(3), 1\right) & \text { for even } k, \\ S_{k}\left(\Gamma_{0}(3), \omega\right) & \text { for odd } k\end{cases}
$$

Now we can apply the Eichler-Selberg trace formula for $S_{k}\left(\Gamma_{0}(3), \chi\right)$, expressing traces of Hecke operators in class numbers of binary quadratic forms.

Proposition 2.1. Let $q=3^{m}$ with $m \geq 1$, and denote by $\operatorname{Tr} T_{q}$ the trace of the Hecke operator $T_{q}$ acting on the space of cusp forms $S_{k}\left(\Gamma_{1}(3)\right)$. Then

$$
\operatorname{Tr} T_{q}= \begin{cases}-\sum_{t} \frac{\rho^{k-1}-\bar{\rho}^{k-1}}{\rho-\bar{\rho}} H\left(t^{2}-4 q\right)-1 & \text { for } k \geq 3, \\ -\sum_{t} \frac{\rho^{k-1}-\bar{\rho}^{k-1}}{\rho-\bar{\rho}} H\left(t^{2}-4 q\right)-1+q & \text { for } k=2 .\end{cases}
$$

The summation variable $t$ runs over $\left\{t \in \mathbf{Z}: t^{2}<4 q\right.$ and $\left.t \equiv 1(\bmod 3)\right\}$. The symbols $\rho$ and $\bar{\rho}$ indicate the zeros of the polynomial $X^{2}-t X+q$, and $H\left(t^{2}-4 q\right)$ is the Kronecker class number of $t^{2}-4 q$.

Proof. We start from the Eichler-Selberg trace formula as stated in [6, Theorem 2.2] and employ it for $S_{k}\left(\Gamma_{0}(3), \chi\right)$, where $\chi=1$ for even $k$ and $\chi=\omega$ for odd $k$. In the notation of [6, Theorem 2.2], the contribution of $A_{1}$ is 0 . As to the contribution of $A_{2}$, we notice that $\mu(t, f, n)=\chi(t)$. It follows that

$$
A_{2}=-\sum_{\substack{t \in \mathbf{Z} \\ t^{2}<4 q, t \equiv 1(\bmod 3)}} \frac{\rho^{k-1}-\bar{\rho}^{k-1}}{\rho-\bar{\rho}} H\left(t^{2}-4 q\right)
$$

by adding together terms with $t \equiv 1(\bmod 3)$ and $t \equiv 2(\bmod 3)$. Furthermore, $A_{3}=-1$ in all cases, and $A_{4}=q$ for $k=2$ and $\chi=1$, while $A_{4}=0$ in the other cases. Altogether, we get the above-mentioned formulas.

The numbers $\left(\rho^{k-1}-\bar{\rho}^{k-1}\right) /(\rho-\bar{\rho})$ are symmetric expressions in $\rho$ and $\bar{\rho}$, so they can be written as polynomials $Q_{k-2}(t, q)$ in $t=\rho+\bar{\rho}$ and $q=\rho \bar{\rho}$. 
We have $Q_{0}(t, q)=1$ and $Q_{1}(t, q)=t$. From $\rho^{k+1}-\bar{\rho}^{k+1}=(\rho+\bar{\rho})\left(\rho^{k}-\bar{\rho}^{k}\right)$ $-\rho \bar{\rho}\left(\rho^{k-1}-\bar{\rho}^{k-1}\right)$ we get the recurrence relation

$$
Q_{k}(t, q)=t Q_{k-1}(t, q)-q Q_{k-2}(t, q) \text { for } k \geq 2 \text {. }
$$

The polynomial $Q_{k}$ is, as a polynomial in $\rho$ and $\bar{\rho}$, homogeneous of degree $k$. Therefore, it is also homogeneous of degree $k$ as a polynomial in $t$ and $q$, provided we assign a weight 1 to the variable $t$ and a weight 2 to the variable $q$. Note that $Q_{k}$ is monic in $t$, and has integer coefficients and terms $q^{r} t^{k-2 r}$, where $0 \leq r \leq[k / 2]$. It follows that we can write

$$
t^{i}=\sum_{\substack{j=0 \\ j \text { even }}}^{i} \lambda_{i, j} Q_{i-j}(t, q) q^{j / 2}
$$

The $\lambda_{i, j} \in \mathbf{Z}$ satisfy

$$
\begin{aligned}
& \lambda_{i, j}=0 \text { for } j \notin\{0,1, \ldots, i\} \text { or } j \text { odd }, \\
& \lambda_{0,0}=\lambda_{1,0}=1
\end{aligned}
$$

while the recurrence relation for $Q_{k}$ induces the recurrence relation

$$
\lambda_{i+1, j}=\lambda_{i, j-2}+\lambda_{i, j} \text {. }
$$

Now we rewrite the expressions for $\operatorname{Tr} T_{q}$ on $S_{k}\left(\Gamma_{1}(3)\right)$ in Proposition 2.1 as

(4) $\operatorname{Tr} T_{q}= \begin{cases}-\sum_{t} Q_{k-2}(t, q) H\left(t^{2}-4 q\right)-1 & \text { for odd } k \text { and even } k \geq 4, \\ -\sum_{t} H\left(t^{2}-4 q\right)-1+q & \text { for } k=2 .\end{cases}$

From the formula for $\operatorname{dim} S_{k}\left(\Gamma_{0}(N), \chi\right)$ in [6] we easily derive:

$$
\operatorname{dim} S_{k}\left(\Gamma_{1}(3)\right)= \begin{cases}\operatorname{dim} S_{k}\left(\Gamma_{0}(3), \omega\right)=[k / 3]-1 & \text { for odd } k, \\ \operatorname{dim} S_{k}\left(\Gamma_{0}(3), 1\right)=[k / 3]-1 & \text { for even } k \geq 4, \\ 0 & \text { for } k=2\end{cases}
$$

Because $\operatorname{dim} S_{2}\left(\Gamma_{1}(3)\right)=0$, one has

$$
\operatorname{Tr} T_{2}=-\sum_{t} H\left(t^{2}-4 q\right)-1+q=0 .
$$

\section{THE WEIGHT DISTRIBUTION OF TERNARY MELAS CODES}

Let $q=3^{m}$ with $m \geq 2$. In [1] we derived the weight distribution of the dual ternary Melas code $M(q)^{\perp}$ :

The nonzero weights of $M(q)^{\perp}$ are $w_{t}=2(q-1+t) / 3$, where $t \in \mathbf{Z}, t^{2}<4 q$, and $t \equiv 1(\bmod 3)$. For $t \neq 1$ the frequency of $w_{t}$ is $(q-1) H\left(t^{2}-4 q\right)$; the weight $w_{1}=2 q / 3$ has frequency $(q-1)\{H(1-4 q)+2\}$.

Using the MacWilliams identities and the Eichler-Selberg trace formula, we obtain an expression for the weight distribution of $M(q)$. We will elaborate the result announced in [1, Theorem 2.3]. 
Theorem 3.1. The number $A_{i}$ of code words of weight $i$ in the Melas code $M(q)$ is given by

$$
\begin{aligned}
q^{2} A_{i}= & \left(\begin{array}{c}
q-1 \\
i
\end{array}\right) 2^{i}+2(q-1) \sum_{s=0}^{i}(-1)^{s}\left(\begin{array}{c}
2 q / 3 \\
s
\end{array}\right)\left(\begin{array}{c}
q / 3-1 \\
i-s
\end{array}\right) 2^{i-s} \\
& -(q-1) \sum_{j=0}^{i} W_{i, j}(q)\left(1+\tau_{j+2}(q)\right),
\end{aligned}
$$

where the polynomials $W_{i, j}(q)$ are defined for $0 \leq j \leq i$ by

$$
\begin{gathered}
W_{0,0}=1, \quad W_{1,0}=0, \quad W_{1,1}=-2, \\
(i+1) W_{i+1, j}=-i W_{i, j}-2 q W_{i, j+1}-2 W_{i, j-1}-2(q-i) W_{i-1, j}
\end{gathered}
$$

(otherwise, the $W_{i, j}$ are 0$)$.

By $\tau_{k}(q)$ we denote for $k \geq 3$ the trace of the Hecke operator $T_{q}$ on $S_{k}\left(\Gamma_{1}(3)\right)$. For convenience we let $\tau_{2}(q)=-q$.

Proof. This proof is a modification of the proof of the analogous theorem in [6]. For $0 \leq i \leq q-1$, let $P_{i}(X)$ be the $i$ th Krawtchouk polynomial

$$
P_{i}(X ; q-1,3)=\sum_{s=0}^{i}(-1)^{s}\left(\begin{array}{c}
X \\
s
\end{array}\right)\left(\begin{array}{c}
q-1-X \\
i-s
\end{array}\right) 2^{i-s} .
$$

These polynomials satisfy the recurrence relation

$$
(i+1) P_{i+1}(X)=(2 q-2-i-3 X) P_{i}(X)-2(q-i) P_{i-1}(X) .
$$

We define $f_{i}(X)=P_{i}(2(q-1+X) / 3)$; then

$$
f_{0}(X)=P_{0}(2(q-1+X) / 3)=1, \quad f_{1}(X)=P_{1}(2(q-1+X) / 3)=-2 X
$$

and the recurrence relation becomes

$$
(i+1) f_{i+1}(X)=(-i-2 X) f_{i}(X)-2(q-i) f_{i-1}(X) .
$$

It follows that $f_{i}(X)$ has degree $i$, and we write

$$
f_{i}(X)=\sum_{k=0}^{i} \pi_{i}(k) X^{k}
$$

Now $\pi_{0}(0)=1, \pi_{1}(0)=0, \pi_{1}(1)=-2$, and from (7) we derive

$$
(i+1) \pi_{i+1}(k)=-i \pi_{i}(k)-2 \pi_{i}(k-1)-2(q-i) \pi_{i-1}(k) .
$$

We define $\pi_{i}(k)=0$ for cases other than $0 \leq k \leq i$. When we apply the MacWilliams identities to $M(q)^{\perp}$ and $M(q)$, we get

$$
q^{2} A_{i}=\sum_{t} \text { frequency }\left(w_{t}\right) P_{i}(2(q-1+t) / 3)+P_{i}(0),
$$

where $t$ runs over $\left\{t \in \mathbf{Z}: t^{2}<4 q\right.$ and $\left.t \equiv 1(\bmod 3)\right\}$. Using the weight distribution of $M(q)^{\perp}$ and the polynomials $f_{i}$ introduced above, we find

$$
\frac{q^{2}}{q-1} A_{i}=\sum_{t} H\left(t^{2}-4 q\right) f_{i}(t)+2 f_{i}(1)+\frac{P_{i}(0)}{q-1} \text {. }
$$


From definition (6) we see that $P_{i}(0)=\left(\begin{array}{c}q-1 \\ i\end{array}\right) 2^{i}$ and

$$
f_{i}(1)=P_{i}\left(\frac{2 q}{3}\right)=\sum_{s=0}^{i}(-1)^{s}\left(\begin{array}{c}
2 q / 3 \\
s
\end{array}\right)\left(\begin{array}{c}
q / 3-1 \\
i-s
\end{array}\right) 2^{i-s} .
$$

From (8) we obtain

$$
\sum_{t} H\left(t^{2}-4 q\right) f_{i}(t)=\sum_{j=0}^{i} \pi_{i}(j) \sum_{t} t^{j} H\left(t^{2}-4 q\right) .
$$

By formula (2) this becomes

$$
\sum_{j=0}^{i} \pi_{i}(j) \sum_{\substack{k=0 \\ k \text { even }}}^{j} \lambda_{j, k} q^{k / 2} \sum_{t} Q_{j-k}(t, q) H\left(t^{2}-4 q\right) .
$$

Using (4) combined with the fact that, according to (5), $\operatorname{Tr} T_{q}=0$ on $S_{2}\left(\Gamma_{1}(3)\right)$, and remembering our convention that $\tau_{2}(q)=-q$, we get

$$
\sum_{j=0}^{i} \pi_{i}(j) \sum_{\substack{k=0 \\ k \text { even }}}^{j} \lambda_{j, k} q^{k / 2}\left(-1-\tau_{j-k+2}(q)\right)
$$

We define $W_{i, j}(q)=\sum_{k=0, k \text { even }}^{i-j} \pi_{i}(k+j) \lambda_{k+j, k} q^{k / 2}$. By changing the horizontal summation in (10) into a diagonal summation, the expression (10) becomes

$$
\sum_{j=0}^{i} W_{i, j}(q)\left(-1-\tau_{j+2}(q)\right)
$$

Putting all this together, we get the announced formula for $q^{2} A_{i}$.

As to the polynomials $W_{i, j}(q)$, we easily see that $W_{0,0}=1, W_{1,0}=0$, and $W_{1,1}=-2$. The recurrence relation for $W_{i, j}$ follows by writing out the definition of $(i+1) W_{i+1, j}$ and using the recurrence relations (9) and (3) for $(i+1) \pi_{i+1}(k+j)$ and $\lambda_{k+j, k}$.

We conclude this section by noticing that to obtain more explicit expressions for $A_{i}$, we have to compute the traces of the Hecke operators $\tau_{k}(q)$. This is the subject of the next two sections.

4. The COMPUTATION OF $\tau_{k}(q)$ FOR $k$ EVEN, $k \geq 4$

As always, we take $q=3^{m}$ with $m \geq 2$. By convention, we have that $\tau_{2}(q)=-q$, while for $k \geq 3$ the trace of the Hecke operator $T_{q}$ acting on the space $S_{k}\left(\Gamma_{1}(3)\right)$ is indicated by $\tau_{k}(q)$. For even $k$, the space $S_{k}\left(\Gamma_{1}(3)\right)=$ $S_{k}\left(\Gamma_{0}(3), 1\right)$ and the theory of newforms of Atkin and Lehner [2] provides us with a decomposition

$$
S_{k}\left(\Gamma_{0}(3), 1\right)=S_{k}\left(\Gamma_{0}(3)\right)^{\text {new }} \oplus S_{k}\left(\Gamma_{0}(3)\right)^{\text {old }},
$$

which is respected by the Hecke operators. The old part is spanned by the forms $f(z)$ and $f(3 z)$, where $f(z)$ runs over a basis of simultaneous eigenforms of $S_{k}\left(\Gamma_{0}(1)\right)=S_{k}\left(\mathrm{SL}_{2}(\mathbf{Z})\right)$. 
Proposition 4.1. On $S_{k}\left(\Gamma_{0}(3)\right)^{\text {old }}$ we have

$$
\begin{aligned}
\operatorname{Tr} T_{1}= & 2 \operatorname{dim} S_{k}\left(\mathrm{SL}_{2}(\mathbf{Z})\right) \\
\operatorname{Tr} T_{3}= & \operatorname{Tr}\left(T_{3} \text { on } S_{k}\left(\mathrm{SL}_{2}(\mathbf{Z})\right)\right) \\
\operatorname{Tr} T_{3^{m}}= & \operatorname{Tr}\left(T_{3^{m}} \text { on } S_{k}\left(\mathrm{SL}_{2}(\mathbf{Z})\right)\right) \\
& -3^{k-1} \operatorname{Tr}\left(T_{3^{m-2}} \text { on } S_{k}\left(\mathrm{SL}_{2}(\mathbf{Z})\right)\right) \text { for } m \geq 2 .
\end{aligned}
$$

Proof. The subspace $S_{k}\left(\Gamma_{0}(3)\right)^{\text {old }}$ is a direct sum of 2-dimensional complex vector spaces with basis $\{f(z), f(3 z)\}$, where $f(z)$ is a simultaneous eigenform for all $T_{n}$ in $S_{k}\left(\mathrm{SL}_{2}(\mathbf{Z})\right)$. The operator $T_{1}$ is the identity map, so

$$
\operatorname{Tr} T_{1}=\operatorname{dim} S_{k}\left(\Gamma_{0}(3)\right)^{\text {old }}=2 \operatorname{dim} S_{k}\left(\mathrm{SL}_{2}(\mathbf{Z})\right) \text {. }
$$

Let $f(z)=\sum_{m=1}^{\infty} a_{m} e^{2 \pi i m z}$; then by applying the formula for $T_{n}$ on $S_{k}\left(\Gamma_{0}(3), 1\right)$ (see [6]) we have

$$
T_{3}(f(z))=\sum_{m \geq 1} a_{3 m} e^{2 \pi i m z},
$$

while on $S_{k}\left(\mathrm{SL}_{2}(\mathbf{Z})\right)$ we have

$$
T_{3}(f(z))=\lambda f(z)=\sum_{m \geq 1} a_{3 m} e^{2 \pi i m z}+3^{k-1} \sum_{m \geq 1} a_{m} e^{3(2 \pi i m z)} .
$$

For $T_{3}$ acting on the 2-dimensional summand $\langle f(z)\rangle \oplus\langle f(3 z)\rangle$, we obtain

$$
T_{3}(f(z))=\lambda f(z)-3^{k-1} f(3 z) \text { and } T_{3}(f(3 z))=f(z) \text {. }
$$

Then on $\langle f(z)\rangle \oplus\langle f(3 z)\rangle$ the operator $T_{3}$ has eigenvalues $\alpha$ and $\beta$ with $\alpha+\beta=\lambda$ and $\alpha \beta=3^{k-1}$. The eigenvalues of $T_{3}$ acting on $S_{k}\left(\Gamma_{0}(3)\right)^{\text {old }}$ are precisely the $\alpha$ and $\beta$ for all possible eigenvalues $\lambda$ of $T_{3}$ acting on $S_{k}\left(\mathrm{SL}_{2}(\mathbf{Z})\right)$. We conclude that

$$
\operatorname{Tr} T_{3}=\sum(\alpha+\beta)=\sum \lambda=\operatorname{Tr}\left(T_{3} \text { on } S_{k}\left(\mathrm{SL}_{2}(\mathbf{Z})\right)\right) \text {. }
$$

From the product formula $T_{n} \cdot T_{m}=\sum_{d \mid m, n} d^{k-1} T_{m n / d^{2}}$ we derive

$$
T_{3^{m}}=T_{3} \cdot T_{3^{m-1}}-3^{k-1} T_{3^{m-2}} \text { for } m \geq 2
$$

on $S_{k}\left(\mathrm{SL}_{2}(\mathrm{Z})\right)$. Thus, the eigenvalue $\lambda_{3^{m}}$ of $T_{3^{m}}$ on $S_{k}\left(\mathrm{SL}_{2}(\mathrm{Z})\right)$ corresponding to $\lambda$ is

$$
\lambda \cdot \lambda_{3^{m-1}}-3^{k-1} \cdot \lambda_{3^{m-2}} \text {. }
$$

While $\lambda=\alpha+\beta$ and $3^{k-1}=\alpha \beta$, it follows by induction that the eigenvalue of $T_{3^{m}}$ on $S_{k}\left(\mathrm{SL}_{2}(\mathrm{Z})\right)$ corresponding to $\lambda=\alpha+\beta$ is $\sum_{i=0}^{m} \alpha^{i} \beta^{m-i}$.

Furthermore, it holds that $T_{3^{m}}=\left(T_{3}\right)^{m}$ on $S_{k}\left(\Gamma_{0}(3), 1\right)$, so $T_{3^{m}}$ has eigenvalues $\alpha^{m}$ and $\beta^{m}$ on $\langle f(z)\rangle \oplus\langle f(3 z)\rangle$. Adding up the relation

$$
\alpha^{m}+\beta^{m}=\sum_{i=0}^{m} \alpha^{i} \beta^{m-i}-\alpha \beta \sum_{i=0}^{m-2} \alpha^{i} \beta^{m-2-i}
$$

for all pieces of $S_{k}\left(\Gamma_{0}(3)\right)^{\text {old }}$, we obtain the stated result for $T_{3^{m}}, m \geq 2$.

Remark 4.2. From the dimension formula [6, Corollary 2.3] we conclude $\operatorname{dim} S_{2}\left(\mathrm{SL}_{2}(\mathbf{Z})\right)=0$,

$$
\operatorname{dim} S_{k}\left(\mathrm{SL}_{2}(\mathbf{Z})\right)= \begin{cases}{[k / 12]} & \text { for } k \neq \equiv 2(\bmod 12), \\ {[k / 12]-1} & \text { for } k \equiv 2(\bmod 12), k \geq 4\end{cases}
$$

Next we derive a formula for $\operatorname{Tr} T_{q}$ on $S_{k}\left(\Gamma_{0}(3)\right)^{\text {new }}$. 
Proposition 4.3. On $S_{k}\left(\Gamma_{0}(3)\right)^{\text {new }}$ we have

$$
\operatorname{Tr} T_{q}= \begin{cases}\operatorname{dim} S_{k}\left(\Gamma_{0}(3)\right)^{\text {new }} \cdot q^{k / 2-1} & \text { for } m \text { even } \\ q^{k / 2-1} & \text { for } m \text { odd, } k \equiv 2,6(\bmod 12), \\ -q^{k / 2-1} & \text { for } m \text { odd, } k \equiv 0,8(\bmod 12), \\ 0 & \text { for } m \text { odd, } k \equiv 4,10(\bmod 12) .\end{cases}
$$

Proof. First we consider $T_{3}$. The eigenvalues of $T_{3}$ acting on $S_{k}\left(\Gamma_{0}(3)\right)^{\text {new }}$ are $\pm 3^{k / 2-1}$ (see [3, Theorem 3]). In order to find the multiplicities of the eigenvalues, we compute

$$
\begin{aligned}
\operatorname{Tr} T_{3} \text { on } S_{k}\left(\Gamma_{0}(3)\right)^{\text {new }} & =\operatorname{Tr} T_{3} \text { on } S_{k}\left(\Gamma_{0}(3), 1\right)-\operatorname{Tr} T_{3} \text { on } S_{k}\left(\Gamma_{0}(3)\right)^{\text {old }} \\
& =\operatorname{Tr} T_{3} \text { on } S_{k}\left(\Gamma_{0}(3), 1\right)-\operatorname{Tr} T_{3} \text { on } S_{k}\left(\mathrm{SL}_{2}(\mathbf{Z})\right) .
\end{aligned}
$$

By the Eichler-Selberg formula we find

$$
\begin{aligned}
\operatorname{Tr} T_{3} & \text { on } S_{k}\left(\Gamma_{0}(3), 1\right) \\
& =-\left\{\frac{\rho_{1}^{k-1}-\bar{\rho}_{1}^{k-1}}{\rho_{1}-\bar{\rho}_{1}} h_{w}(-11)+\frac{\rho_{2}^{k-1}-\bar{\rho}_{2}^{k-1}}{\rho_{2}-\bar{\rho}_{2}} h_{w}(-8)+1\right\},
\end{aligned}
$$

where $\rho_{1}, \bar{\rho}_{1}$ are the zeros of $X^{2}-X+3$ and $\rho_{2}, \bar{\rho}_{2}$ are the zeros of $X^{2}-2 X+3$. Applying the same formula for $\operatorname{Tr} T_{3}$ on $S_{k}\left(\mathrm{SL}_{2}(\mathrm{Z})\right)$, we find (13) and the extra terms

$$
-\left(\frac{\rho_{3}^{k-1}-\bar{\rho}_{3}^{k-1}}{\rho_{3}-\bar{\rho}_{3}} h_{w}(-3)\right)-\frac{1}{2}\left(\frac{\rho_{4}^{k-1}-\bar{\rho}_{4}^{k-1}}{\rho_{4}-\bar{\rho}_{4}}\right)\left(h_{w}(-12)+h_{w}(-3)\right),
$$

where $\rho_{3}, \bar{\rho}_{3}$ are the zeros of $X^{2}-3 X+3$ and $\rho_{4}, \bar{\rho}_{4}$ are the zeros of $X^{2}+3$. For $\Delta<-4$, the $h_{w}(\Delta)$ are class numbers and $h_{w}(-3)=1 / 3$.

Note that in the case of $\mathrm{SL}_{2}(\mathbf{Z})$, the character involved is the principal character, which is 1 on all of $\mathbf{Z}$ and has conductor 1 .

Substituting the zeros of $X^{2}-3 X+3$ and $X^{2}+3$, we get

$$
\begin{aligned}
\operatorname{Tr} T_{3} \text { on } S_{k}\left(\Gamma_{0}(3)\right)^{\text {new }} & =2.3^{k / 2-2}(\sin (k-1) \pi / 6+\sin (k-1) \pi / 2) \\
& = \begin{cases}0 & \text { for } k \equiv 4,10(\bmod 12), \\
3^{k / 2-1} & \text { for } k \equiv 2,6(\bmod 12), \\
-3^{k / 2-1} & \text { for } k \equiv 0,8(\bmod 12) .\end{cases}
\end{aligned}
$$

Denoting the multiplicities of the eigenvalues $3^{k / 2-1}$ and $-3^{k / 2-1}$ by $A$ and $B$, respectively, we now know $A-B$, while $A+B=\operatorname{dim} S_{k}\left(\Gamma_{0}(3)\right)^{\text {new }}$. Because $T_{3^{m}}=\left(T_{3}\right)^{m}$ on $S_{k}\left(\Gamma_{0}(3), 1\right)$, the eigenvalues of $T_{3^{m}}$ on $S_{k}\left(\Gamma_{0}(3)\right)^{\text {new }}$ are $\left(3^{k / 2-1}\right)^{m}$ and $\left(-3^{k / 2-1}\right)^{m}$, while their multiplicities are known as well. From (14) we easily confirm the required result.

The dimension of $S_{k}\left(\Gamma_{0}(3)\right)^{\text {new }}$ for even $k \geq 4$ can be computed explicitly. From the decomposition

$$
S_{k}\left(\Gamma_{0}(3), 1\right)=S_{k}\left(\Gamma_{0}(3)\right)^{\text {new }} \oplus S_{k}\left(\Gamma_{0}(3)\right)^{\text {old }}
$$

we see that $\operatorname{dim} S_{k}\left(\Gamma_{0}(3)\right)^{\text {new }}=\operatorname{dim} S_{k}\left(\Gamma_{1}(3)\right)-2 \operatorname{dim} S_{k}\left(\mathbf{S L}_{2}(\mathbf{Z})\right)$. Combining 
(5) and (12), one finds

$$
\operatorname{dim} S_{k}\left(\Gamma_{0}(3)\right)^{\text {new }}=2[k / 12]+ \begin{cases}-1 & \text { for } k \equiv 0(\bmod 12), \\ 1 & \text { for } k \equiv 2,6,8(\bmod 12), \\ 0 & \text { for } k \equiv 4(\bmod 12), \\ 2 & \text { for } k \equiv 10(\bmod 12) .\end{cases}
$$

Conclusion. To calculate $\tau_{k}(q)$ for $k$ even, $k \geq 4$, and $q=3^{m}, m \geq 2$, we put Propositions 4.1 and 4.3 and formula (15) for $\operatorname{dim} S_{k}\left(\Gamma_{0}(3)\right)^{\text {new }}$ together. The only quantity left over to compute is the trace of $T_{q}$ on $S_{k}\left(\mathrm{SL}_{2}(\mathbf{Z})\right)$. In the next proposition we include a small list of traces of Hecke operators $\tau_{k}(q)$ for even $k$.

Proposition 4.4. The trace $\tau_{k}(q)$ of the Hecke operator $T_{q}$, where $q=3^{m}$ with $m \geq 1$, acting on $S_{k}\left(\Gamma_{1}(3)\right)$, is for even $k$ satisfying $4 \leq k \leq 22$ given by the following table:

$\begin{array}{rccc}k & \tau_{k}(q) \text { on } S_{k}\left(\Gamma_{0}(3)\right)^{\text {old }} & \tau_{k}(q) \text { on } S_{k}\left(\Gamma_{0}(3)\right)^{\text {new }} \\ & & m \text { odd } & m \text { even } \\ 4 & 0 & 0 & 0 \\ 6 & 0 & q^{2} & q^{2} \\ 8 & 0 & -q^{3} & q^{3} \\ 10 & 0 & 0 & 2 q^{4} \\ 12 & t_{12, m} & -q^{5} & q^{5} \\ 14 & 0 & q^{6} & 3 q^{6} \\ 16 & t_{16, m} & 0 & 2 q^{7} \\ 18 & t_{18, m} & q^{8} & 3 q^{8} \\ 20 & t_{20, m} & -q^{9} & 3 q^{9} \\ 22 & t_{22, m} & 0 & 4 q^{10}\end{array}$

For $k=12,16,18,10,22$, the $t_{k, m}$ are respectively given by $t_{k, 0}=2, t_{k, 1}=$ $252,-3348,-4284,50652,-128844$ and

$$
t_{k, m}=t_{k, 1} \cdot t_{k, m-1}-3^{k-1} t_{k, m-1} \text { for } m \geq 2 .
$$

Proof. For $k=4,6,8,10,14$, the spaces $S_{k}\left(\mathrm{SL}_{2}(\mathbf{Z})\right)$ are zero, therefore $\operatorname{Tr} T_{q}$ on $S_{k}\left(\Gamma_{0}(3)\right)^{\text {old }}$ is zero, and our formulas follow easily.

For $k=12,16,18,20,22$, the spaces $S_{k}\left(\mathrm{SL}_{2}(\mathrm{Z})\right)$ are one-dimensional. If $\lambda$ is the eigenvalue of $T_{3}$ on $S_{k}\left(\mathrm{SL}_{2}(\mathrm{Z})\right)$, we have $\lambda=\alpha+\beta$ and $3^{k-1}=\alpha \beta$, where $\alpha$ and $\beta$ are the corresponding eigenvalues of $T_{3}$ on $S_{k}\left(\Gamma_{0}(3)\right)^{\text {old }}$ (see the proof of Proposition 4.1). Now $t_{m}=\operatorname{Tr} T_{3^{m}}$ on $S_{k}\left(\Gamma_{0}(3)\right)^{\text {old }}$ satisfies the recurrence relation

$$
t_{m}=\alpha^{m}+\beta^{m}=\lambda t_{m-1}-3^{k-1} t_{m-2} \text { for } m \geq 2,
$$


while $t_{1}=\lambda$ and $t_{0}=2$. We calculate $\lambda=\operatorname{Tr} T_{3}$ on $S_{k}\left(\mathrm{SL}_{2}(\mathbf{Z})\right)$ by the trace formula. The result is

$$
\lambda=-\sum_{t=0}^{3} r_{t} Q_{k-2}(t, 3)-1
$$

with $r_{0}=\frac{2}{3}, r_{1}=r_{2}=1$, and $r_{3}=\frac{1}{3}$. Combining these observations with Proposition 4.3, we obtain our formulas.

Note that for $k=12$, the eigenvalue of $T_{q}$ on $S_{12}\left(\mathrm{SL}_{2}(\mathbf{Z})\right)$ is $\tau(q)$, where $\tau$ is the Ramanujan $\tau$-function. Then $t_{m}=\tau(q)-3^{11} \tau(q / 9)$ for $q=3^{m}$ with $m \geq 2$ and $t_{1}=252$.

\section{THE COMPUTATION OF $\tau_{k}(q)$ FOR ODD $k \geq 3$}

By (5), we have for odd $k$ that

$$
\operatorname{dim} S_{k}\left(\Gamma_{1}(3)\right)=\operatorname{dim} S_{k}\left(\Gamma_{0}(3), \omega\right)=[k / 3]-1 .
$$

Since the action of the character $\omega$ on $(Z / 3 Z)^{*}$ differs from the action of the principal character, the space of cusp forms $S_{k}\left(\Gamma_{0}(3), \omega\right)$ consists entirely of newforms. Therefore, the eigenvalues $\lambda$ of $T_{3}$ acting on $S_{k}\left(\Gamma_{0}(3), \omega\right)$ have absolute values $3^{(k-1) / 2}$ (see [3, Theorem 3]). This implies that the monic polynomial $F_{k}(X)$ with roots $\lambda / 3^{(k-1) / 2}$ is reciprocal. So, to determine $F_{k}(X)$, which has degree $[k / 3]-1$, we only have to know the first $[([k / 3]-1) / 2]+1$ coefficients, provided they are not 0 .

Since $T_{q}=\left(T_{3}\right)^{m}$ on $S_{k}\left(\Gamma_{0}(3), \omega\right)$, we have $\operatorname{Tr} T_{q}=\sum_{\lambda} \lambda^{m}$, and from the Newton identities for power sums we can derive some elementary symmetric functions of the eigenvalues $\lambda$ from $\operatorname{Tr} T_{3}, \operatorname{Tr} T_{9}$, etc. We only need a few $\operatorname{Tr} T_{q}$ to fix $F_{k}(X)$, bearing in mind that $F_{k}(X)$ is reciprocal. From $F_{k}(X)$ we obtain the characteristic polynomial of $T_{3}$ and from that the eigenvalues $\lambda$ of $T_{3}$. Then we can compute $\tau_{k}(q)=\sum_{\lambda} \lambda^{m}$ for odd $k \geq 3$ and $q=3^{m}$ with $m \geq 2$.

Proposition 5.1. The trace $\tau_{k}(q)$ of the Hecke operator $T_{q}$ with $q=3^{m}$ and $m \geq 2$, acting on $S_{k}\left(\Gamma_{0}(3), \omega\right)$, is for $k=3,5,7,9,11,13,15$, and 17 given by the following table:

$$
\begin{aligned}
\tau_{3}(q) & =\tau_{5}(q)=0, \\
\tau_{7}(q) & =(-1)^{m} q^{3}, \\
\tau_{9}(q) & =q^{4} \cdot \operatorname{Trace}\left(\alpha_{9}^{m}\right), \\
\tau_{11}(q) & =q^{5} \cdot \operatorname{Trace}\left(\alpha_{11}^{m}\right), \\
\tau_{13}(q) & =q^{6} \cdot\left\{1+\operatorname{Trace}\left(\alpha_{13}^{m}\right)\right\}, \\
\tau_{15}(q) & =q^{7} \cdot \operatorname{Trace}\left(\alpha_{15}^{m}\right), \\
\tau_{17}(q) & =q^{8} \cdot \operatorname{Trace}\left(\alpha_{17}^{m}\right) .
\end{aligned}
$$


The $\alpha_{i}$ are algebraic numbers of absolute value 1 given by

$$
\begin{gathered}
\alpha_{9}=\frac{5+2 \sqrt{-14}}{9}, \quad \alpha_{11}=\frac{-1+4 \sqrt{-5}}{9}, \quad \alpha_{13}=\frac{-25+2 \sqrt{-26}}{27}, \\
\alpha_{15}=\frac{61-16 \sqrt{91}+4 \sqrt{-2002-122 \sqrt{91}}}{243}, \\
\alpha_{17}=\frac{-19+2 \sqrt{8089}+2 \sqrt{-6583-19 \sqrt{8089}}}{243} .
\end{gathered}
$$

In this table, the Trace of an algebraic number is the sum of all its conjugates.

Proof. Since $\operatorname{dim} S_{k}\left(\Gamma_{0}(3), \omega\right)=0$ for $k=3,5$, we have that $\tau_{3}(q)=\tau_{5}(q)=$ 0 . For the other values of $k$ we compute the eigenvalues of $T_{3}$ acting on $S_{k}\left(\Gamma_{1}(3)\right)=S_{k}\left(\Gamma_{0}(3), \omega\right)$ in the way indicated above. The trace formula (4) gives us that for weight $k$ :

$$
\begin{aligned}
\operatorname{Tr} T_{3} & =-Q_{k-2}(1,3) H(-11)-Q_{k-2}(-2,3) H(-8)-1 \\
& =-Q_{k-2}(1,3)-Q_{k-2}(-2,3)-1, \\
\operatorname{Tr} T_{9} & =-1-\sum_{\substack{t \equiv 1(\bmod 3) \\
t^{2}<36}} Q_{k-2}(t, 9) H\left(t^{2}-36\right) .
\end{aligned}
$$

Using the recurrence relations for the polynomial $Q_{k-2}$ and a small table of class numbers from [7], we get the entries of the table below:

$$
\begin{array}{rrrl}
k & \operatorname{Tr} T_{3} & \operatorname{Tr} T_{9} & \multicolumn{1}{c}{F_{k}(X)} \\
7 & -27 & 729 & X+1 \\
9 & 90 & -5022 & X^{2}-\frac{10}{9} X+1 \\
11 & -54 & -115182 & X^{2}+\frac{2}{9} X+1 \\
13 & -621 & 1291059 & (X-1)\left(X^{2}+\frac{50}{27} X+1\right) \\
15 & 2196 & -1624860 & X^{4}-\frac{244}{243} X^{3}+\frac{1474}{2187} X^{2}-\frac{244}{243} X=1 \\
17 & -2052 & 18618660 & X^{4}+\frac{76}{243} X^{3}-\frac{122}{729} X^{2}+\frac{76}{243} X+1
\end{array}
$$

The eigenvalues $\lambda$ of $T_{3}$ on $S_{k}\left(\Gamma_{0}(3), \omega\right)$ are $3^{(k-1) / 2} \cdot \alpha$, where $\alpha$ runs over the zeros of $F_{k}(X)$ and

$$
\tau_{k}(q)=\sum_{\lambda} \lambda^{m}=q^{(k-1) / 2} \sum_{\left\{\alpha: F_{k}(\alpha)=0\right\}} \alpha^{m},
$$

which provides us with our formulas for $k=7,9,11,13,15,17$.

Note that by computing the trace of more $T_{3^{m}}$ for $m \geq 3$ we can easily extend Proposition 5.1. Adding $\operatorname{Tr} T_{27}$, for instance, will get us to $k=23$.

\section{WeIGHT FORMULAS FOR $M(q)$}

When we combine Theorem 3.1 with Propositions 4.4 and 5.1, we get explicit formulas for the frequencies $A_{i}$ of words of weight $i$ in $M(q)$. To obtain these formulas, we used the symbolic manipulation language MACSYMA. 
We conclude by giving a table of weight formulas. In this table, Ramanujan's $\tau$-function is denoted by $\tau$ and the numbers $t_{k}$ denote $\operatorname{Trace}\left(\alpha_{k}^{m}\right)$ as in Proposition 5.1 .

\section{TABLE 6.1}

Frequencies $A_{i}$ of small weights $i$ in the Melas codes $M(q)$

$$
\begin{aligned}
& A_{1}=A_{3}=0, \\
& A_{2}=q-1 \text {, } \\
& A_{4}=(q-1)(q-3) / 2 \text {, } \\
& A_{5}=4(q-1)\left(q^{2}+\left((-1)^{m}-14\right) q+36\right) / 15 \text {, } \\
& A_{6}=(q-1)\left(8 q^{3}-165 q^{2}+\left(1240-68(-1)^{m}\right) q-2655\right) / 90 \text {, } \\
& A_{7}=2(q-1)\left(4 q^{4}-108 q^{3}+\left(4 t_{9}-18(-1)^{m}+1215\right) q^{2}\right. \\
& \left.+\left(399(-1)^{m}-6744\right) q+12884\right) / 315, \\
& A_{8}=(q-1)\left(16 q^{5}-560 q^{4}+8225 q^{3}-\left(224 t_{9}-880(-1)^{m}+66255\right) q^{2}\right. \\
& \left.-\left(16296(-1)^{m}-298263\right) q-517825\right) / 2520 \text {, } \\
& A_{9}=(q-1)\left(16 q^{6}-704 q^{5}+13216 q^{4}\right. \\
& -\left(160 t_{9}-16 t_{11}-216(-1)^{m}+138656\right) q^{3} \\
& +\left(3816 t_{9}-13776(-1)^{m}+895209\right) q^{2} \\
& \left.-\left(3470238-187593(-1)^{m}\right) q+5597820\right) / 11340 \text {, } \\
& A_{10}=(q-1)\left(32 q^{7}-1728 q^{6}+40512 q^{5}-540519 q^{4}\right. \\
& +\left(6240 t_{9}-720 t_{11}-6120(-1)^{m}+4529826\right) q^{3} \\
& +\left(-110280 t_{9}+360000(-1)^{m}-24851277\right) q^{2} \\
& +\left(85643448-4448871(-1)^{m}\right) q-129806479 \\
& \left.-32(\tau(q)-177147 \tau(q / 9)) / q^{2}\right) / 113400, \\
& A_{11}=(q-1)\left(32 q^{8}-2080 q^{7}+59520 q^{6}-985920 q^{5}\right. \\
& +\left(2288 t_{9}+32 t_{13}-560 t_{11}-440(-1)^{m}+10453958\right) q^{4} \\
& +\left(-136840 t_{9}+16720 t_{11}+110220(-1)^{m}-74203966\right) q^{3} \\
& +\left(1705506 t_{9}-5122359(-1)^{m}+358627785\right) q^{2} \\
& +\left(57077625(-1)^{m}-112429735\right) q+1617492524 \\
& \left.+880(\tau(q)-177147 \tau(q / 9)) / q^{2}\right) / 623700, \\
& A_{12}=(q-1)\left(64 q^{9}-4928 q^{8}+168960 q^{7}-3400320 q^{6}\right. \\
& +44564751 q^{5}-\left(2112 t_{13}-33440 t_{11}+115808 t_{9}\right. \\
& \left.+398775397+16720(-1)^{m}\right) q^{4}-\left(664400 t_{11}\right. \\
& \left.-5020400 t_{9}+2939640(-1)^{m}-2486674179\right) q^{3} \\
& -\left(52961436 t_{9}-145879734(-1)^{m}+10845159710\right) q^{2} \\
& +\left(31412188148-1550485266(-1)^{m}\right) q-43190708055 \\
& \left.+(\tau(q)-177147 \tau(q / 9))\left(1408 q^{4}-46992 q^{3}\right) / q^{5}\right) / 7484400 \text {. }
\end{aligned}
$$




\section{BIBLIOGRAPHY}

1. G. van der Geer and M. van der Vlugt, Artin-Schreier curves and codes, J. Algebra 139 (1991), 256-272.

2. S. Lang, Introduction to modular forms, Grundlehren der Math. Wiss., no. 222, SpringerVerlag, New York, 1976.

3. W. W. Li, Newforms and functional equations, Math. Ann. 212 (1975), 285-315.

4. J. MacWilliams and N. J. A. Sloane, The theory of error-correcting codes, North-Holland, Amsterdam, 1983.

5. C. M. Melas, A cyclic code for double error correction, IBM J. Res. Develop. 4 (1960), 364-366.

6. R. Schoof and M. van der Vlugt, Hecke operators and the weight distributions of certain codes, J. Combin. Theory Ser. A 57 (1991), 163-186.

7. R. Schoof, Non-singular plane cubic curves over finite fields, J. Combin. Theory Ser. A 46 (1987), 183-211.

8 J.-P. Serre, Cours d'arithmétique, Presses Univ. de France, Paris, 1970.

Faculteit Wiskunde en Informatica, Universiteit van Amsterdam, 1018 TV AmsterDAM, THE NETHERLANDS

E-mail address: geer@fwi.uva.nl

Dipartimento di Matematica, Università di Trento, 38050 Povo (Trento), Italy

E-mail address: schoof@itnvax.cineca.it

Afdeling Wiskunde en Informatica, Universiteit van Leiden, 2300 Ra Leiden, The NetherLANDS 